\title{
VERITAS: Mind-mapping in Virtual Reality
}

\author{
Robert Sims \\ School of Computing and \\ Communicatuions \\ Lancaster University \\ Lancaster, United Kingdom \\ r.sims2@lancaster.ac.uk
}

\author{
Abhijit Karnik \\ School of Computing and \\ Communicatuions \\ Lancaster University \\ Lancaster, United Kingdom \\ a.karnik@lancaster.ac.uk
}

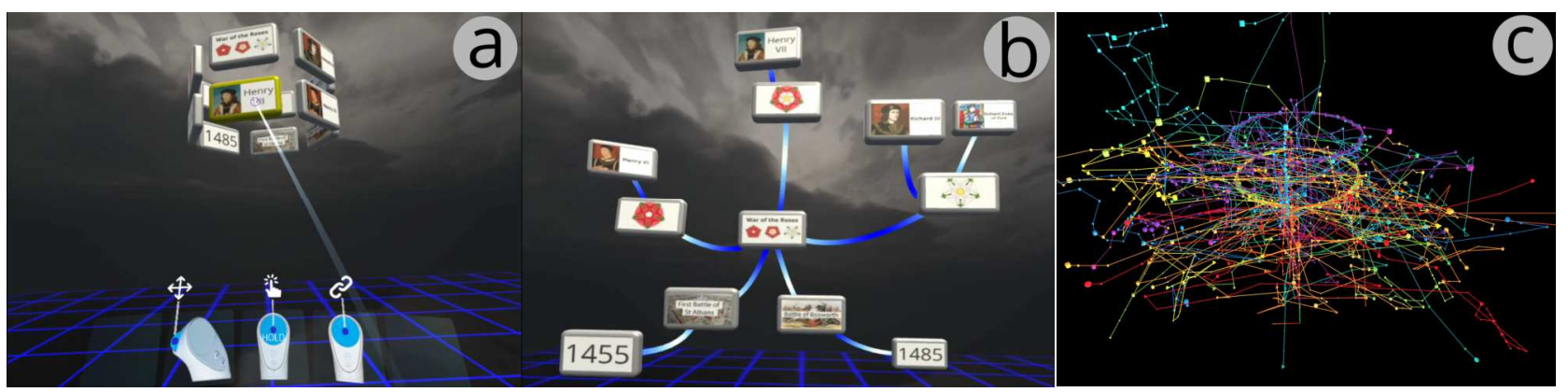

Fig. 1. Mind-mapping using VERITAS. (a) Initial 'carousel' of interactive tiles. (b) Completed "War of Roses" mind-map with animated links showing directional relationships. (c) Visualization of tile movements by users showing tile movements.

\begin{abstract}
Inquiry based learning is a modern and innovative learning strategy that aims to stimulate students' interest in a topic and target Bloom's higher order cognitive process. Reflective tasks, such as mind mapping, support inquiry-based learning. Virtual Reality (VR) presents novel opportunities to help scaffold reflective tasks in inquiry-based learning by supporting use of the 3D space which is not available via existing 2D mind mapping applications. In this paper we present VERITAS, a VR application for mind-mapping based reflective tasks operating on the low-cost Oculus Go device. We discuss the interaction design for the mindmapping task and evaluate the system from a usability perspective. Our results show that novice participants are able to learn how to use the interactions quickly and utilize them effectively to build mind-maps in 3D. VERITAS establishes the usability of VR and essential interactions to successfully perform abstract and complex reflective tasks like mind-mapping.
\end{abstract}

Index terms-Virtual Reality, Engagement, Education, Reflective tasks, Inquiry-based Learning

\section{INTRODUCTION}

VR (Virtual Reality) has the potential to significantly impact education and specifically students' engagement in the learning process [1]-[3]. Recent advances in VR technology have made low-cost untethered VR headsets accessible to more users. Low cost VR devices such as the Oculus Go are untethered and consequently more manageable in a traditional classroom environment. Due to the untethered nature, these devices also present the intriguing possibility of being included by educational institutions in their flipped learning strategy [4].

Beyond the novelty factor of VR headsets, it is essential to understand the exact use which benefits the learning process. Commercially, low-cost devices are geared toward content consumption rather than content creation. Educational institutions commonly use VR as exploration devices, such as viewing 360-degree videos of interesting places on earth, visualizing chemical structures or viewing parts of the galaxy. These activities are typically passive in nature, with limited interactivity and as such address only the lower cognitive processes, such as those illustrated in Blooms Taxonomy [5] of remembering and understanding. Conversely, inquiry based learning [6] incorporates reflective tasks such as categorising, organising, differentiation and interpretation. The aim is to trigger the more advanced cognitive processes of applying and analysis. Examples of reflective tasks currently used within education include white boarding and mind maps [7], [8]. These reflective tasks are known to show benefits over teacher-led learning [9]. However, in the VR domain there are very few applications that support interactive reflection. For VR and consequently our developed application, VERITAS, these tasks are particularly interesting as they present an opportunity to explore abstractly structured reflection within a 3D spatial environment. We focus on individual reflection and the role of VR in supporting this process as a starting point for investigating VR supported reflection in paragogy (peer-based learning).

The main contributions of this paper are:

- The concept and analysis of a VR mind mapping application to be used as a scaffolding tool for inquirybased learning.

- The implementation and analysis of specific complex interactions required for the VR mind mapping application.

- The results of a user experience study and discussion on the learnability of the application. 


\section{BACKGROUND}

\section{A. Reflective Tasks and Inquiry-based Learning}

Inquiry-based learning forms one of many innovative modern approaches to learning. Kirschner et al. [10] state that inquiry based learning is key to stimulating students' desire to learn more and discover information semi-independently of the teacher and/or classroom. It promotes the ability to construct ways of presenting discovered information and a way to reflect on the effectiveness of discovering and presenting new information. To make this minimally guided learning strategy effective, sound scaffolding strategies need to be implemented [11]-[15]. Scaffolding allows learners to engage in tasks that otherwise might be too complex for them to manage given their current abilities. Examples of scaffolding include undertaking reflective tasks, such as mind mapping, diagramming or diary keeping and using technology-based solutions, such as applications and constructing websites to display information.

Inquiry-based learning relies on interest or active engagement in a cognitive exercise [16]. Interest plays a core part in regulating our emotional engagement in undertaking a task [17], what we choose to learn [18], and the efficacy of learning that information [19]. Situational interest arises from the appeal of the actual activity to the learner rather than their predisposition towards the topic [16], [20]. When designing educational tools, situational interest is a key consideration as it also develops personal interest for learners and is critical to positively enhance learning [21]. Sotiriou and Bogner [22] identified that Augmented Reality (AR), a technology closely related to VR, can elevate students' interest and motivation, leading to them developing enhanced investigation skills while gaining topic knowledge.

With VERITAS, we explore how to best exploit VR as a reflective task while simultaneously providing a scaffolding tool for teachers that assists the inquiry process for students, promotes situational interest and aids the overall learning experience.

\section{B. Mind-mapping and Virtual Reality}

Virtual reality is well-established as an educational tool throughout a broad range of fields. These tools operate as a custom environment and require interactions to simulate realworld tasks. For example, VR educational tools in the medical field allow clinical protocols to be simulated, practiced [23] and assessed [24] risk-free, thus enhancing student learning. Similar results are observed in engineering by Kaufmann et al. [25], where students found the Construct3D tool easy and quick to learn and appreciated the tool's ability to let them experiment with their ideas. There are demonstrable advantages of supporting reflection to explore a specialized topic but limited to an environment similar to where the knowledge would be applied.

Mind-maps are an educational tool that allow learners to offset difficulties commonly ascribed to natural limitations with working memory and its capacity. Recalling and managing disparate elements of information are recognized as learning tasks with a high cognitive load [26]. Mind-maps can alleviate this cognitive load by allowing the learner to interact with a graphical representation of ideas and relationships. Mind-maps are implemented as an abstraction of the knowledge from the environment of application, cognitively a step closer to how the mind organizes the information than how the information is applied. The simplest form of the mind-map tool is a pen and paper activity which can be extended to a white-board and sticky notes. The information is organized in a $2 \mathrm{D}$ planar space with interconnecting links formed between related keywords. The digital form of this activity has been explored in the context of: information organization [27]; collaborative thinking [28], [29] and as a research tool to understand collaboration between young learners [30].

VR presents a unique opportunity for mind-mapping exercises as it can inherently support spatial organization of information in 3D. Within $\mathrm{AR}$, the existence of virtual objects has already been demonstrated to assist students in visualizing abstract concepts and complex visual relationships [31]. VR can also support interesting interactions and collaboration, acting as a "one-world multiple-perspectives" environment for exploration of concepts. As a learning tool in the classroom, it provides unique opportunities for the educator to direct and shape the conversation around a pre-determined set of keywords while allowing unique behaviours and interaction strategies to emerge among the learners.

Digital implementations of mind-mapping are criticised for being slower than traditional pen and paper mind mapping, often turning into an exercise in tool management, rather than spending time on the actual core mind mapping activity itself [32]. The challenge for VR is to identify efficient and intuitive ways of using VR concepts to create mind maps. VR based mind-mapping is less understood and very few examples [33] are available. The open question is how to converge the existing concepts of VR-based interaction into a fluid interaction experience such that the focus of the user is on reflection and abstract thinking arising from mind-mapping rather than wielding of the tools to operate within the VR environment.

VERITAS aims to address this challenge by focusing on designing and evaluating a mind-mapping experience for a single user by leveraging existing validated VR interaction paradigms.

\section{Virtual Reality and Interaction Design}

High-fidelity interaction with virtual environments is still an active area of research despite spanning many decades. Early research [34] identified that users have difficulty understanding three-dimensional space. Interaction recommendations from these early studies included using ray casting for target acquisition coupled with a 'silk cursor' to provide feedback and context to the user [35]. Further research [36] identified design strategies to allow users to estimate size and distance within virtual worlds by way of providing landmarks and reference objects that act as visual clues. With the recent advances in virtual reality hardware in terms of both headsets and controllers, recent research has centered on implementing real word interactions within the virtual world [37]-[39]. However, there is an inherent risk that the designed interaction can end up being worse than low fidelity interactions [40]. The balance between implementing natural, low fidelity and metaphorical interactions is an important factor in the user experience associated with VR application design. VERITAS leverages well-known interaction techniques while trying to understand their effect on user 
experience when these are integrated into a single interaction workflow.

Feedback is another important consideration within interaction design for virtual environments. Haptic feedback for hand motions [41], [42] can assist in immersion but requires special hardware. Immersion can be also enhanced by audio cues in digital experiences [43]. Sound plays a critical role in providing essential feedback to the user, such as providing warnings, indicating errors or confirmation of user actions [44], [45] where sound quality has greater effect than image quality on presence [46]. Low-cost commercial VR headsets generally lack high-fidelity haptic feedback controllers but support higher quality sound. With VERITAS, we choose to exploit audio feedback to augment visual feedback for specific notifications during interaction.

The most common physiological issue with VR is simulator sickness resulting from visually induced motion. Hettinger et al. [47] identified that vection and motion sickness can occur at the same time and further studies have shown strong links between both [48]-[50]. Vection is even more pronounced in VR systems that only employ 3DoF (Degrees of Freedom) versus a $6 \mathrm{DoF}$ through their hardware. VERITAS uses a seated experience with no sudden in-application locomotion to reduce simulator sickness by minimizing vection.

\section{SYSTEM DESIGN}

\section{A. VR Platform}

The motivation for VERITAS is a classroom-based setting where reflective tasks like mind-mapping are to be carried out. This presents constraints related to choices like tethering, tracking and control in addition to unit cost and supporting infrastructure. A pilot survey of available hardware indicated that the entire spectrum supported 3DoF controller input at minimum with additional features like clickable buttons, swipe surfaces or joystick alternatives. This formed our baseline for selecting our test hardware. While 6DoF controllers with higher fidelity exist, the interactions we explore can only be further improved by $6 \mathrm{DoF}$ (when such become a low-cost option), while continuing to work on existing hardware. The Oculus Go controller includes a touch surface which can interpret swipe gestures in the form of thumb swipe up, down, left and right in order to expand the possible interactions available to the application. The controller also includes a gyroscope for $3 \mathrm{DoF}$ input and interactions were built around this also. As a low-cost untethered unit, the Oculus Go acts a flexible VR platform for implementation. VERITAS is implemented using the Unity game development platform and the Oculus Integration framework (v1.35). To retain full control of interaction development, no other VR toolkits were used. The application is forward compatible with Oculus Rift and Quest series of headsets.

\section{B. Implementation}

\section{1) System Overview}

The primary design goal of VERITAS is to allow participants to build a mind map from pre-defined objects based on information they had previously been exposed to outside of VERITAS. These pieces of unique information could be images or text and needed to be presented as interactive objects. From an interaction perspective, participants can manipulate and arrange these objects and display relationships between these objects. The information objects are represented as double-sided tiles. A tile carries the same content (image or text) on both sides. The tiles are initially presented as a rotating carousel (Fig. 1a) so users can see all available tiles before deciding on which tile to interact with. Relationships between objects are represented by a curvilinear link object. The link connects a parent and child tile and a pulsing animation is applied to the link to show this 'fromto' relationship. The links are designed to redraw themselves as the connected tiles are moved.

The working volume is a $10 \times 10 \times 10$ unit cube ( 1 Unity unit $\approx 1$ metre), bounded by a floor with a grid pattern and transparent walls on the remaining 5 sides. A neutral skybox is applied to the entire scene. The tile carousel is located at $(0,2.5,1)$. A model representing the physical controller and the controlling hand is displayed on the lower half of the viewing frustum. The model mirrors any change along the $3 \mathrm{DoF}$ as well as button clicks and swipes on the touch-pad. Further feedback of the interaction state is provided using a heads-up display (HUD) panel at the lower edge of the view. It shows actions available to the user in the current interaction state as well as highlighting the actions as they are performed. These elements are shown in Fig. $1 \mathrm{a}, 2 \mathrm{a}, 2 \mathrm{~b}$ and $2 \mathrm{c}$.

\section{2) Interaction Workflow}

The interaction workflow is based on a state-model approach. The start point is a controller in tracking mode waiting for target selection. The tracking is in the form of a ray-cast pointer emanating from the controller model. The direction vector for the ray is determined by the orientation of the controller. When the ray intersects a tile, the tile is highlighted yellow (Fig. 1a). Once a tile is selected, the highlight color changes to green (Fig. 2a) and the interactions available for the tile are linked to the controller inputs. The HUD updates to show what actions are available.

The user can perform the default action or enter a deeper interaction mode as required. In all cases, the 'Back' controller button reverts the user to the prior state without undoing any previously completed actions. We provided a full set of audio cues and notification prompts for confirmation of actions and invalid actions.

We took certain design decisions based on pilot tests of particular interactions and situations that could arise during the use of the mind-map. For example, tiles cannot occupy the same $3 \mathrm{D}$ space. If a user tries to position an object into a location which is already occupied by another object, the selected object will slide over, under or to the side of the existing object in the direction of its trajectory.

To aid operation while only using a 3DoF controller, the tile translation action was separated into planar and depth movement. For the planar motion (Fig. 2a), once selected the user can move the tile up-down and right-left but keep the tile at the same fixed distance from themselves thus moving the tile on a cylindrical curved surface. The decoupled depth motion is available as an independent interaction using a push-pull metaphor through up-down swipe gestures on the touchpad producing movement along the z-axis (away from or towards the user). These interactions are placed orthogonally to the scale interactions (swipe right-left). This placement was intended to 

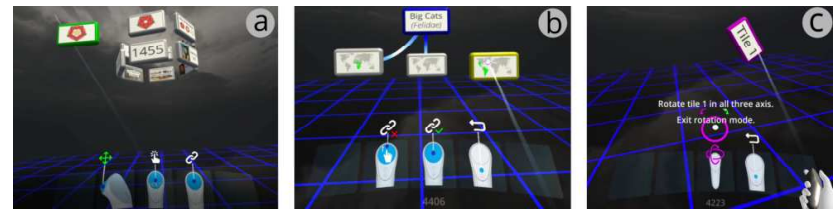

Fig. 2. Interactions for mind-mapping a) Moving a tile out of the carousel (tile highleted green), b) creating links between tiles (tile highlighted blue), c) rotating a tile (tile highlited purple).

accentuate the difference between the two interactions as both produce visually similar outcomes from the perspective of the user.

For rotation, the naïve approach of linking the controller orientation to rotation was quickly discarded. In pilot tests, this approach was extremely difficult to control and angles exceeding 30 degrees were hard to achieve without uncomfortable contortion of the wrist. Instead, we chose to use a hysteresis-based implementation (Fig. 2c). Once the rotation state is entered, any change in the controller orientation is ignored till it exceeds a threshold angle value. Once exceeded, the tile rotation is then controlled by coupling the tile rotation rate to the controller orientation angle along the specific axis. This is analogous to a steering wheel of a car and it prevented users from accidentally triggering rotation before they were ready to perform the action. The HUD's feedback was analogous to a crossing-based interactive widget [51] (Fig. 2c).

The link creation interaction is tied to tiles. The user can enter the link creation state once a tile is highlighted. The highlighted tile is treated as the parent tile (Fig. 2b). The user then points to the child tile and selects it to complete the link between the two. Deletion similarly starts by highlighting the parent tile and entering the delete-link state.

\section{3) Simulator Sickness Considerations}

Simulator sickness resulting from visually induced motion is a common and well-studied physiological issue associated with VR [47]. Due to the disparity between the users' vestibular and visual systems, vection is even more pronounced in VR systems that only support $3 \mathrm{DoF}$ versus $6 \mathrm{DoF}$ through their hardware [52], [53]. VERITAS is a seated experience with no sudden inapplication locomotion or change in the position of the user. This alleviates simulator sickness by minimizing vection.

\section{EXPERIMENT}

The mind-mapping task supported by VERITAS is wellknown in inquiry-based learning approaches. The individual VR interactions implemented in VERITAS are based on existing literature. However, the motivation of the experiment is to understand if the interaction workflow can allow a user to focus on the mind-mapping activity instead of focusing on tool management. We would also like to understand if the interaction workflow can be learnt quickly enough by novice users. Finally, as an exploration of mind-mapping in 3D, we wish to identify the emergence of interesting interaction patterns that can inform future work in a collaborative context. With this in mind, we conducted a study.

\section{A. Apparatus}

We used an Oculus Go stand-alone VR headset for the study. The default factory settings were retained for the purpose of the study, including interpupillary distance, brightness and volume.
The headset was configured to store the desired logging information and videos.

\section{B. Participants}

24 participants over 18 years of age were selected from Lancaster University and Blackpool and the Fylde College to participate in the study. Participants did not require prior experience of virtual reality and there were no stipulated exclusion criteria that would prevent potential participants taking part in the study. The experiment was conducted after acquiring the requisite ethical approvals from Lancaster University.

Our participant sample included twenty males, four females, with ages ranging from 18 to 50 years of age. Fifteen of participants were from the 18-25 age-range. Four participants had no prior VR experience, while one participant had used VR headsets only once before.

\section{Task}

We chose three unrelated topics to explore the mindmapping exercise - the animal kingdom, a web technology hierarchy and a branching history timeline. The topic, selected via a round robin approach, was presented to the participants as a one-page document containing information related to the topic. We then setup a mind-mapping exercise in VERITAS for each of these topics. The exercise consisted of keywords and pictures, with pictures either representing physical entities (i.e., animals, people or objects) or illustrative entities (i.e., maps, actions or symbols). Keywords also included dates and numerical values. An example of a completed activity based on one of the above three scenarios can be seen in Fig. $1 b$.

The tasks consisted of a pre-activity, the main task and a post activity. The pre-activity task and post-activity task were simple instruction tasks that asked the participant to perform interactive actions like scaling a tile and linking two tiles. The participant had to complete each interaction step before moving to the next task. The required steps were the same for both activities. The main activity consisted of an open-ended exploration of the topic as a mind-mapping exercise. The participants were instructed to build the mind-map based on the text they had read and reproduce the relationships between the tiles as best as they could. Once they were satisfied with the mind-map, they could signal completion.

\section{Metrics}

Participants' activities within the system were recorded by way of a video screen capture and system logging that recorded different parameters.

All interactions using the controller were logged along with the context within the interaction workflow. We treated inputs in an incorrect context as errors and logged these. For example, attempting to select a tile when none was highlighted would be treated as an error. We also logged the position, orientation and size of all the tiles on a periodic basis. The video feed of the VR space was captured to obtain a participant view of what was visible on the headset.

Participants completed a standardized User Experience Questionnaire (UEQ), a standard Simulator Sickness Questionnaire (SSQ) and were given an opportunity to provide 


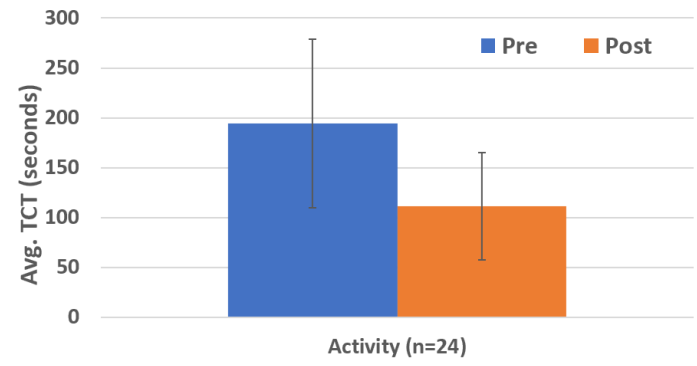

Fig. 3. Pre and post activity completion times.

open-ended feedback about their experience. The UEQ is a widely used tool for measuring user experience and the quality of applications. The UEQ is designed to elicit a quick and spontaneous response regarding the application or product being assessed and generates statistics for six elements attractiveness, perspicuity, efficiency, dependability, stimulation and novelty. It also includes a benchmark for comparison against existing applications [54]. A well-designed application is expected to score positively on the UEQ. Scores in the range of +2 represent a very positive result. Negative scores indicate poor user experience with the application. Extreme scores above +2 are rarely returned due to the common occurrence of respondents avoiding answering at the extreme ends of scales. The UEQ has a high internal consistency [55].

\section{E. Procedure}

A repeated measures within-subjects design was used. Each participant was given a pre-selected topic to ensure equal participation for each topic. They participated in the experiment in one continuous session lasting up to 25 minutes plus 5 minutes to complete questionnaires. Before starting the tasks, the participants undertook a short tutorial inbuilt to the device to familiarize themselves with the headset and controls. The preactivity task and the post-activity task were identical in structure. For the post-activity we removed the helper tips that were provided to assist in performing the interaction (e.g., 'hold button B to select an object' for the pre-activity versus 'select an object' for the post-activity). This tested the ability of the participants to recall how to effectively interact with the application and undertake all the required interactions without instruction. We expected that there would be a reduction in interaction errors and a shorter activity completion time for the post-activity versus the pre-activity task.

The UEQ generates statistics for six elements attractiveness, perspicuity, efficiency, dependability, simulation and novelty. It also includes a benchmark for comparison against existing applications [54]. However, the benchmark is based on non-VR applications it should be noted that dataset used to construct the benchmark does not specifically include other VR applications. Overall, a well-designed application could be expected to score positively on the UEQ.

\section{RESULTS}

The logged data was analyzed for the following: pre-post activity completion times, interaction errors, UEQ, SSQ and free responses.

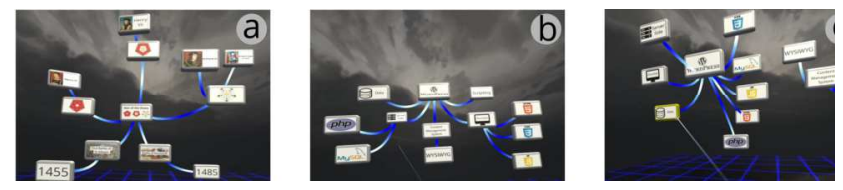

Fig. 4. Hierarchical organization styles used by participants, (a) Radial, (b) Tree and (c) star.

\section{A. Pre/Post Activity Completion Times}

For the Pre and Post activity completion times (TCT) we performed an ANOVA with repeated measures. There was a statistically significant difference in TCT between the Pre and Post activity tasks $(\mathrm{F}(1,23)=33.07, \mathrm{p}<.05)$ with the Post activity $(\mu \mathrm{Po}=111.54 \mathrm{~s})$ being completed significantly faster than the Pre activity $(\mu \mathrm{Pr}=194.54 \mathrm{~s})$.

The individual comparison of Pre and Post task completion times is shown in Fig. 3. There's a clear trend of reduced completion time in post activity tasks.

\section{B. Interaction Errors}

We analyzed the error rates for each interaction category (translate, select, rotate and link) and using an ANOVA with repeated measures test, found no significant difference between the error rates for both pre-activity and post-activity tasks. Overall, we observed that the error rates were very low $(\mu 0.31 \%$ for pre-activity and $\mu 0.68 \%$ for post-activity) in all categories.

\section{Main Activity Analysis}

We observed that every user successfully created a clear and recognizable mind map with complete relationships and good spatial positioning, including in the z-axis. They made full use of all the available interactions to manipulate the tiles and build their mind-map. We observed that completed mind-maps followed one of three styles - radial, tree or star (see Fig. 4), with radial being the most common style with twelve occurrences, seven for tree and five for star.

Quantitatively, we also looked at error rates, how users utilized the 3D space and how long they took to complete the main activity task. We noted that most users made use of the zaxis in some dimension ( $\mu 3.58$ unity units) and the overall error rates were low ( $\mu 0.97 \%$ or all interactions). The average time to complete the main activity task was 398 s with no outliers. We explored the possibility that the topic selected for the mind-map activity could present itself as an experimental confound. To eliminate this, we used one-way ANOVA, with 'topic' as the between-subjects factor for analysis against error rate, activity completion times and tile movement and found no statistically significant difference to suggest that the topic was a factor.

\section{D. $U E Q$}

The UEQ is provided with an automated analysis tool for generating results. Results from the tool are reported here. The participants scored VERITAS high in terms of attractiveness, stimulation and novelty (see Fig. 5). The scores for perspicuity, efficiency and dependability were also positive but lower. Expert VR users could bias the Hedonic Quality metric of UEQ. However, previous work [55]-[57] does not discuss the bias as a factor affecting UEQ scores for familiar users (mere-exposure effect versus expertise). Since none of our participants identified 


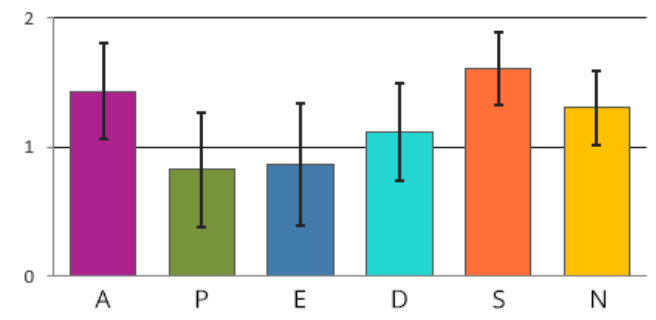

Fig. 5. UEQ Scales $A=$ Attractiveness, $P=$ Perspicuity, $E=$ Efficiency, $D$ $=$ Dependability, $\mathrm{S}=$ Stimulation, $\mathrm{N}=$ Novelty. UEQ scale range is $[-3,3]$ but is truncated due to absence of negative values.

as "Expert", we approach the question of bias in line with previous work.

\section{E. $S S Q$}

Responses to SSQ showed no notable increase in discomfort or any form of nausea. Only one participant noted an increase in discomfort (pins and needles in hands). The same participant noted existing issues with fine motor control for their thumb.

\section{F. User Responses}

The participants were asked to reflect upon their experience in an open-ended format. Seven participants noted that the HUD location was too low in the viewport and one didn't notice the HUD at all. Two participants stated they felt there were too many steps required to delete a link. Three participants stated they would like to be able to select multiple objects and move them as a group.

\section{G. Visualisation}

Using the logged tile position data, we created a 3D visualization to explore tile movements (Fig. 1c shows a composite of five participants tile movements) during the main activity. The plot displays the movement of every tile for each user. The time ( $t)$ spent by a tile at each location is represented by a shape enclosed in a sphere of diameter $=\log 10 \mathrm{t}$. This plot shows that participants used the full space afforded by the virtual environment for creating a mind-map.

\section{DISCUSSION}

Our aim was to investigate if a simple yet usable interaction workflow could be developed for interacting with a mind-map in VR.

\section{A. Usability of VERITAS}

The quantitative analysis of the Pre/Post Activity tasks is the first indicator for the usability of VERITAS. We find an expected reduction in task completion times (TCT) for the posttask. Apart from learning effect, the positive observation of low error rates indicates that the participants were able to work with the interaction metaphors without extra effort. If the interaction metaphors were harder to recognize or recall, the absence of the helper prompts in the post-activity would have affected the TCT or error rates, which was not the case. All users completed the main activity to a reasonable standard of completeness and quality, indicating that all the essential interactions are in place.

The main activity and completeness of the resulting mind maps demonstrated that the participants were able to understand and use the state-model for the interaction workflow with relative ease. Even if some interactions (like rotation) were placed deeper than the default state, the participants navigated the states without difficulty. This state-model is supported by low-cost $3 \mathrm{DoF}$ controllers that are commonly available. However, if $6 \mathrm{DoF}$ controllers become commonplace, the statemodel can be mapped to the controller inputs with minimal modifications.

The UEQ provides further insight into the usability. The strong results for attractiveness and novelty metrics would be normally expected for a well-designed VR application. The stimulation metric indicates higher motivation to continue using the product. This observation is particularly important as situational interest is essential to inquiry-based learning and strengthens the argument for performing mind-mapping exercises in VR. Perspicuity, efficiency and dependability metrics are positive though not as strong as the prior metrics. The perspicuity metric measures the pragmatic qualities and learnability of the application. The metrics indicate that users found the application to be a different learning experience than usual.

The SSQ responses indicate that our design choices did not result in increased discomfort while using the application. The feedback from the participants were mainly positive. One participant mentioned that the interactions appeared to be a blend of intuitive versus learnable. The participant with high select and rotate errors mentioned that they had an issue with fine-motor control of their thumb. This led to accidental clicking of the touchpad when trying to swipe, causing selection and rotate errors. The participant also noted that they were able to recover from the accidental inputs and continue successfully without much effort. This raises a point for consideration about accessibility of controllers but is beyond the scope of current research.

\section{B. Mind-mapping in $V R$}

The motivation for VERTIAS was to identify a suitable interaction workflow to support creation of mind-maps in VR. We also wished to see how users would make use of the 3D space available to them for organizing the information. The video analysis and the tile position visualizations showed a very good use of 3D space with each participant producing a clearly identifiable mind-map in one of three styles. Participants made ample use of the spatial positions, logging interactions to move the tiles back and forth along the Z-axis (depth interaction). They also focused on the orienting the tiles to suit a view that they preferred. The use of the $Z$ axis suggests that mind mapping in VR may offer advantages over traditional 2D implementations.

The participants were able to understand and use the statemodel for the interaction workflow with relative ease. Even if some interactions (like rotation) were several layers deep from the default state, the participants navigated this without difficulty. This state-model is supported by low-cost $3 \mathrm{DoF}$ controllers that are commonly available. However, if $6 \mathrm{DoF}$ controllers become commonplace, the state-model can be mapped to the controller inputs with minimal modifications.

\section{Design Discussion}

The interaction workflow of VERITAS is designed for use with low-cost 3DoF controllers. While we ran the studies on an Oculus Go headset, VERITAS could be easily ported to a low- 
fidelity smartphone setup (e.g., Cardboard) making it even more accessible or to the Oculus Quest for increased interactions possibilities afforded by a $6 \mathrm{DoF}$ headset and controllers.

While the current scope of VERITAS is a single-user mindmap activity, the leap to a collaborative mind-mapping application is obvious. When collaborative mind-mapping is carried out on tabletops, the collaborative exercise results in specific patterns of communication and strategies for managing conflict [58]. These arise due to the need to control shared pieces of information (e.g., tiles) and their relative positions. Users do try to move objects at the same time; however, users will relinquish control of an object if they perceive another's actions is correct (termed collaborative interplay). Collaborative mindmapping in VR has the opportunity to support richer interactions and collaborations since the independent headsets can support 'one-world, multiple perspectives' for the task. Collaborative strategies involving shared workspaces and personal workspaces [59] can be applied to VR with relative ease while continuing to foster communication between the peers. A key to enabling effective collaboration within VR is the need for communicating system state of all objects (i.e., an item being moved) to all users and that the users' actions are apparent to all other users (i.e., gaze directions, pointer direction, if they are modifying an object).

With the usability of VERITAS established, exploration of user behaviors within the application is required to develop the application further to support collaborative learning. There is also the obvious question - Does learning indeed occur within a VR mind mapping application (and how to measure such learning)? The current design of VERITAS provides a baseline system that can be expanded in the future and trivially instrumented to further understand collaborative interactions as well as evaluation of learning effectiveness and user mindmapping strategies.

\section{CONCLUSION}

This study demonstrated that low-cost devices, in spite of their limited input controls, are capable of supporting complex reflective task activities, specifically in the form of mind mapping. The required interactions were complete and minimal interaction errors were generated. An important factor to consider is the learnability of such applications. While interactions can be complex, supporting them through means such as audio feedback and a UI to communicate system state to the user can create an environment where a user can learn how the system functions and their performance improves within the system over time. Furthermore, there does appear to be an advantage in conducting a mind mapping activity within VR as several participants utilized the full 3D spatial area that VR affords to manipulate their mind map in ways not traditionally available in existing 2D applications.

\section{REFERENCES}

[1] E. A. O'Connor and J. Domingo, "A Practical Guide, With Theoretical Underpinnings, for Creating Effective Virtual Reality Learning Environments," J. Educ. Technol. Syst., vol. 45, no. 3, pp. 343-364, 2017, doi: $10.1177 / 0047239516673361$.

[2] D. Parmar et al., "Programming moves: Design and evaluation of applying embodied interaction in virtual environments to enhance computational thinking in middle school students," in 2016 IEEE Virtual
Reality (VR), 2016, vol. 2016-July, pp. 131-140, doi: 10.1109/VR.2016.7504696.

[3] A. A. Rizzo et al., "The Virtual Classroom: A Virtual Reality Environment for the Assessment and Rehabilitation of Attention Deficits," CyberPsychology Behav., vol. 3, no. 3, pp. 483-499, 2002, doi: 10.1089/10949310050078940.

[4] J. Abdullah, W. N. Mohd-Isa, and M. A. Samsudin, "Virtual reality to improve group work skill and self-directed learning in problem-based learning narratives," Virtual Real., no. 0123456789, 2019, doi: 10.1007/s10055-019-00381-1.

[5] S. Bloom, Taxonomy of Educational Objectives. New York: Mckay, 1956.

[6] M. Pedaste et al., "Phases of inquiry-based learning: Definitions and the inquiry cycle," Educ. Res. Rev., vol. 14, pp. 47-61, 2015, doi: 10.1016/j.edurev.2015.02.003.

[7] I. Abi-El-Mona and F. Adb-El-Khalick, "The Influence of Mind Mapping on Eighth Graders' Science Achievement," Sch. Sci. Math., vol. 108, no. 7, pp. 298-312, 2008, doi: 10.1111/j.1949-8594.2008.tb17843.x.

[8] C. J. Wenning, "Whiteboarding and Socratic dialogues: Questions and answers," J. Phys. Teach. Educ. Online, vol. 3, no. 1, pp. 3-10, 2005.

[9] T. S. Shin, J. Ranellucci, and C. J. Roseth, "Effects of peer and instructor rationales on online students' motivation and achievement," Int. J. Educ. Res., vol. 82, pp. 184-199, 2017, doi: 10.1016/j.ijer.2017.02.001.

[10] P. A. Kirschner, J. Sweller, and R. E. Clark, "Why Minimal Guidance During Instruction Does Not Work: An Analysis of the Failure of Constructivist, Discovery, Problem-Based, Experiential, and InquiryBased Teaching," Educ. Psychol., vol. 41, no. 2, pp. 75-86, 2006, doi: 10.1207/s15326985ep4102_1.

[11] A. Collins, J. S. Brown, and A. Holum, "Cognitive apprenticeship: Making thinking visible," Am. Educ., vol. 15, no. 3, pp. 6-11, 1991.

[12] R. Golan, E. A. Kyza, B. J. Reiser, and D. C. Edelson, "Scaffolding the Task of Analyzing Animal Behavior with the Animal Landlord Software," Annu. Meet. Am. Educ. Res. Assoc., pp. 1-24, 2002.

[13] S. L. Jackson, S. J. Stratford, J. Krajcik, and E. Soloway, "Making Dynamic Modeling Accessible to Precollege Science Students," Interact. Learn. Environ., vol. 4, no. 3, pp. 233-257, 1994, doi: $10.1080 / 1049482940040305$.

[14] W. A. Sandoval and B. J. Reiser, "Explanation-driven inquiry: Integrating conceptual and epistemic scaffolds for scientific inquiry," Sci. Educ., vol. 88, no. 3, pp. 345-372, 2004, doi: 10.1002/sce.10130.

[15] E. E. Toth, D. D. Suthers, and A. M. Lesgold, “'Mapping to know': The effects of representational guidance and reflective assessment on scientific inquiry," Sci. Educ., vol. 86, no. 2, pp. 264-286, Mar. 2002, doi: $10.1002 /$ sce. 10004 .

[16] G. Schraw and S. Lehman, "Situational Interest: A Review of the Literature and Directions for Future Research Author ( s )," Rev. Educ. Psychol., vol. 13, no. 1, pp. 23-52, 2014, doi: 10.1023/A:1009004801455.

[17] U. Schiefele, "Interest and Learning From Text," Sci. Stud. Read., vol. 8438 , no. 1999, pp. 37-41, 2009, doi: 10.1207/s1532799xssr0303_4.

[18] S. E. Wade, G. Schraw, W. M. Buxton, and M. T. Hayes, "Seduction of the Strategic Reader: Effects of Interest on Strategies and Recall," Read. Res. Q., vol. 28, no. 2, p. 92, 1993, doi: 10.2307/747885.

[19] P. A. Alexander and T. L. Jetton, "The role of importance and interest in the processing of text," Educ. Psychol. Rev., vol. 8, no. 1, pp. 89-121, 1996, doi: 10.1007/BF01761832.

[20] L. Linnenbrink-Garcia et al., "Measuring situational interest in academic domains," Educ. Psychol. Meas., vol. 70, no. 4, pp. 647-671, 2010, doi: 10.1177/0013164409355699.

[21] S. Hidi and K. Ann Renninger, "The four-phase model of interest development," Educ. Psychol., vol. 41, no. 2, pp. 111-127, 2006, doi: $10.1207 / \mathrm{s} 15326985 \mathrm{ep} 4102 \_4$.

[22] S. Sotiriou and F. X. Bogner, "Visualizing the Invisible: Augmented Reality as an Innovative Science Education Scheme," Adv. Sci. Lett., vol. 1, no. 1, pp. 114-122, 2008, doi: 10.1166/asl.2008.012.

[23] R. S. Haluck, "Computers and Virtual Reality for Surgical Education in the 21st Century," Arch. Surg., vol. 135, no. 7, p. 786, 2000, doi: 10.1001/archsurg.135.7.786. 
[24] W. English, "Virtual reality and surgical education.," J. Surg. Simul., vol. 5, no. 2-2, 2000, doi: 10.1102/2051-7726.2018.a002.

[25] Kaufmann H. Schmalstieg D. Wagner M., "Construct3D: A Virtual Reality Application for Mathematics and Geometry Education," Educ. Inf. Technol., vol. 5, no. 4, p. 14, 2000, doi: 10.1023/A:1012049406877.

[26] S. Tergan, "Digital Concept Maps for Managing Knowledge and Information," in Conference: Knowledge and Information Visualization, Searching for Synergies, 2005, pp. 185-204.

[27] J. Beel and S. Langer, "An exploratory analysis of mind maps," in Proceedings of the 11th ACM symposium on Document engineering, 2011, p. 81, doi: 10.1145/2034691.2034709.

[28] H. Faste and H. Lin, "The untapped promise of digital mind maps," in Proceedings of the 2012 ACM annual conference on Human Factors in Computing Systems - CHI '12, 2012, p. 1017, doi: $10.1145 / 2207676.2208548$.

[29] H. Lin and H. Faste, "Digital Mind Mapping: Innovations for Real-time Collaborative Thinking," CHI'11 Ext. Abstr. Hum. Factors Comput. Syst., pp. 2137-2142, 2011, doi: 10.1145/1979742.1979910.

[30] I. Jamil, K. O'Hara, M. Perry, A. Karnik, and S. Subramanian, “The effects of interaction techniques on talk patterns in collaborative peer learning around interactive tables," in Proceedings of the 2011 annual conference on Human factors in computing systems - CHI '11, 2011, p. 3043, doi: $10.1145 / 1978942.1979393$.

[31] T. N. Arvanitis et al., "Human factors and qualitative pedagogical evaluation of a mobile augmented reality system for science education used by learners with physical disabilities," Pers. Ubiquitous Comput., vol. 13, no. 3, pp. 243-250, 2009, doi: 10.1007/s00779-007-0187-7.

[32] V. Chik, B. Plimmer, and J. Hosking, "Intelligent Mind-mapping," in Conference: Proceedings of the 2007 Australasian Computer-Human Interaction Conference, 2007, pp. 195-198, doi: $10.1145 / 1324892.1324931$.

[33] Coding Leap LLC, "NODA.” 2019, [Online]. Available: http://noda.io/.

[34] K. Hinckley, R. Pausch, J. C. Goble, and N. F. Kassell, "A survey of design issues in spatial input," in Proceedings of the 7th annual ACM symposium on User interface software and technology - UIST '94, 1994, pp. 213-222, doi: 10.1145/192426.192501.

[35] S. Zhai, W. Buxton, and P. Milgram, "The 'Silk Cursor,"” in Proceedings of the SIGCHI conference on Human factors in computing systems celebrating interdependence - CHI '94, 1994, pp. 459-464, doi: $10.1145 / 191666.191822$.

[36] N. G. Vinson, "Design guidelines for landmarks to support navigation in virtual environments," in Proceedings of the SIGCHI conference on Human factors in computing systems the CHI is the limit - CHI '99, 1999, no. May, pp. 278-285, doi: 10.1145/302979.303062.

[37] C. Lai, R. P. McMahan, and J. Hall, "March-and-Reach: A realistic ladder climbing technique," 2015 IEEE Symp. 3D User Interfaces, 3DUI 2015 Proc., pp. 15-18, 2015, doi: 10.1109/3DUI.2015.7131719.

[38] R. P. McMahan et al., "Evaluating natural interaction techniques in video games," 3DUI 2010 - IEEE Symp. 3D User Interfaces 2010, Proc., pp. 11-14, 2010, doi: 10.1109/3DUI.2010.5444727.

[39] M. Nabiyouni, A. Saktheeswaran, D. A. Bowman, and A. Karanth, "Comparing the Performance of Natural, Semi-Natural, and NonNatural," IEEE Virutal Real. 2015, pp. 243-244, 2015, doi: doi:10.1109/3dui.2015.7131717.

[40] R. P. McMahan, C. Lai, and S. K. Pal, Virtual, Augmented and Mixed Reality, vol. 10280. Cham: Springer International Publishing, 2017.

[41] H. Benko, C. Holz, M. Sinclair, and E. Ofek, "NormalTouch and TextureTouch," in Proceedings of the 29th Annual Symposium on User Interface Software and Technology, 2016, pp. 717-728, doi: $10.1145 / 2984511.2984526$.

[42] M. Azmandian, M. Hancock, H. Benko, E. Ofek, and A. D. Wilson, "A Demonstration of Haptic Retargeting," in Proceedings of the 2016 ACM on Interactive Surfaces and Spaces, 2016, no. May, pp. 501-504, doi: $10.1145 / 2992154.2996883$.

[43] I. Ekman, "On the desire to not kill your players: Rethinking sound in pervasive and mixed reality games.," Ext. Abstr. Publ. Annu. Symp. Comput. Interact. Play, pp. 142-149, 2013.

[44] C. Johanson and R. L. Mandryk, "Scaffolding Player Location Awareness through Audio Cues in First-Person Shooters," Proc. 2016 CHI Conf. Hum. Factors Comput. Syst., pp. 3450-3461, 2016, doi: $10.1145 / 2858036.2858172$.

[45] K. Jørgensen, "Left in the dark: playing computer games with the sound turned off," in From Pac-Man to Pop Music, K. Collins, Ed. Farnham: Routledge, 2017, pp. 163-176.

[46] J. J. Cummings and J. N. Bailenson, "How Immersive Is Enough? A MetaAnalysis of the Effect of Immersive Technology on User Presence," Media Psychol., vol. 19, no. 2, pp. 272-309, 2016, doi: 10.1080/15213269.2015.1015740.

[47] L. J. Hettinger, K. S. Berbaum, R. S. Kennedy, P. Dunlap, and M. D. Nolan, "Vection and Simulator Sickness," Mil. Psychol., vol. 5605, no. March 2012, pp. 37-41, 2009, doi: 10.1207/s15327876mp0203_4.

[48] G. C. H. Lee, Y. Yoo, and S. Jones, "Investigation of driving performance, vection, postural sway, and simulator sickness in a fixed-based driving simulator," Comput. Ind. Eng., vol. 33, no. 3-4, pp. 533-536, Dec. 1997, doi: 10.1016/S0360-8352(97)00186-1.

[49] S. Classen, M. Bewernitz, and O. Shechtman, "Driving simulator sickness: An evidence-based review of the literature," Am. J. Occup. Ther., vol. 65, no. 2, pp. 179-188, 2011, doi: 10.5014/ajot.2011.000802.

[50] L. Stoffregen, T; Smart, "Postural instability precedes motion sickness," Brain Res. Bull., vol. 47, no. 5, pp. 437-448, 1997.

[51] J. Accot and S. Zhai, "More than dotting the i's --- foundations for crossing-based interfaces," Proc. SIGCHI Conf. Hum. factors Comput. Syst., no. 4, p. 73, 2002, doi: 10.1145/503376.503390.

[52] E. M. Kolasinski, "Simulator Sickness in Virtual Environments," United States Army Research Institute for the Behavioral and Social Sciences, vol. 1027, no. 4. Alexandria, VA, p. 68, 1995, doi: 10.1121/1.404501.

[53] J. J. LaViola, "A discussion of cybersickness in virtual environments," ACM SIGCHI Bull., vol. 32, no. 1, pp. 47-56, 2000, doi: $10.1145 / 333329.333344$.

[54] M. Schrepp, A. Hinderks, and J. Thomaschewski, "Construction of a Benchmark for the User Experience Questionnaire (UEQ)," Int. J. Interact. Multimed. Artif. Intell., vol. 4, no. 4, p. 40, 2017, doi: 10.9781/ijimai.2017.445.

[55] H. B. Santoso, M. Schrepp, R. Yugo Kartono Isal, A. Y. Utomo, and B. Priyogi, "Measuring user experience of the student-centered E-learning environment," J. Educ. Online, vol. 13, no. 1, pp. 1-79, 2016.

[56] B. Laugwitz, T. Held, and M. Schrepp, "Construction and Evaluation of a User Experience Questionnaire," in Proceedings of the 4th Symposium of the Workgroup Human-Computer Interaction and Usability Engineering of the Austrian Computer, 2008, pp. 63-76, doi: 10.1007/978-3-54089350-9_6.

[57] J. Hinderks, A; Schrepp, M; Thomaschewski, "User Experience Questionnaire (UEQ)," 2018. https://www.ueq-online.org (accessed Sep. 24, 2020).

[58] I. Jamil et al., "Collaborating around Digital Tabletops," ACM Trans. Comput. Interact., vol. 24, no. 3, pp. 1-30, May 2017, doi: $10.1145 / 3058551$.

[59] A. Karnik, D. Martinez Plasencia, W. Mayol-Cuevas, and S. Subramanian, "PiVOT," in Proceedings of the 25th annual ACM symposium on User interface software and technology - UIST '12, 2012, p. 271 , doi: $10.1145 / 2380116.2380151$. 